\title{
TRATAMIENTO DE LA INESTABILIDAD VESICAL (VEJIGA HIPERACTIVA NO NEURÓGENA) EN NIÑOS, CON TOLTERODINA*
}

\author{
J.M. GARAT BARREDO, J. CAFFARATTI SFULCINI, E. DE LA PEÑA \\ Unidad de Urología Pediátrica. Servicio de Urología. Fundació Puigvert. Barcelona.
}

Actas Urol Esp. 28 (2): 122-128, 2004

\section{RESUMEN}

TRATAMIENTO DE LA INESTABILIDAD VESICAL (VEJIGA HIPERACTIVA NO NEURÓGENA) EN NIÑOS, CON TOLTERODINA* INTRODUCCIÓN: La vejiga hiperactiva (VHA) se engloba dentro de las disfunciones miccionales no neurógenas, cuya prevalencia ha sido bien definida en la población general, no así en la edad pediátrica.

Las manifestaciones clínicas son variadas y tiene especial importancia en los niños su asociación con otras patologías como la enuresis, el reflujo vésico-ureteral (RVU) y las infecciones de repetición.

El tratamiento fundamental se realiza con fármacos anticolinérgicos. La eficacia del cloruro de oxibutinina ha sido suficientemente contrastada, sin embargo la dosificación y los efectos secundarios, aunque escasos en los niños, son motivo de interrupción del tratamiento. OBJETIVOS: Como alternativa para el tratamiento de la VHA se ha utilizado la tolterodina en adultos con eficacia, sin embargo su uso no ha sido suficientemente valorado en niños.

Deseamos determinar la eficacia y tolerabilidad de dicho fármaco en la población infantil afecta de VHA.

MATERIAL Y MÉTODOS: Estudio retrospectivo de 72 niños diagnosticados de VHA no neurógena y virgenes de tratamiento.

En todos los casos se practicó un protocolo diagnóstico de patología urológica concomitante así como estudio urodinámico (EUD) y exploración neurológica.

En un grupo de estos pacientes se realizó EUD post-tratamiento.

RESULTADOS: La edad media fue de 10,9 años y fueron evaluados entre 4 y 31 meses tras iniciar el tratamiento.

Hubo curación demostrada con cistomanometría en el $67 \%$ de los casos, $14 \%$ mejoraron y un $19 \%$ no presentó variación en el EUD. Según los criterios del International Children's Continence Society (ICCS) aplicados a los niños sin EUD post-tratamiento, el 51\% se curaron, un $27 \%$ mejoró y finalmente un $22 \%$ no tuvo mejoría.

Ningún paciente tuvo que suspender el tratamiento por efectos secundarios.

CONCLUSIONES: La tolerancia y efectividad de la tolterodina es buena en la población pediátrica y constituye una alternativa a los clásicos anticolinérgicos en el tratamiento de la VHA.

PALABRAS CLAVE: Hiperactividad vesical. Tolterodina. Edad infantil.

\section{ABSTRACT}

TREATMENT OF VESICAL INSTABILITY (NO NEUROGENIC OVERACTIVE BLADDER) WITH TOLTERODINA IN CHILDREN INTRODUCTION: Overactive bladder (OB) is one of the no-neurogenic voiding dysfunctions whose prevalence has been precisely defined among the general population but not so among the paediatric population.

Its clinical manifestations are various, and its association with other pathologies like enuresis, vesico-ureteral reflux (VUR) and recurrent infections is particularly significant in children.

OB is basically managed with anticholinergic drugs. The efficacy of oxybutynin chloride has been sufficiently proved; however its dosage and side effects, although scarce in children, usually cause treatment discontinuation.

OBJECTIVES: Tolterodine has been successfully used as an alternative therapy of OB in adults, however its use has not been sufficiently evaluated in children.

Our objective is to determine tolterodine's efficacy and tolerability in the paediatric population suffering from OB.

MATERIAL AND METHODS: A retrospective study of 72 children who were diagnosed no-neurogenic OB and who received no previous treatment.

A concomitant urological pathology diagnostic protocol was applied to all cases, as well as a urodynamic test (UDT) and a neurological examination.

Post-treatment UDT was performed to one group of patients

RESULTS: The mean age was 10.9 years and the children were assessed between 4 and 31 months after treatment initiation.

Healing was proved through cistomanometry in $67 \%$ of the cases, there was improvement in $14 \%$ and $19 \%$ of the patients showed no changes in the UDT.

Following the criteria of the International Children's Continence Society (ICCS) applied to those children with no post-treatment UDT, 51\% were healed, $27 \%$ improved and $22 \%$ experienced no changes.

None of the patients had to discontinue the treatment due to side effects.

CONCLUSIONS: Tolterodine's tolerability and efficacy are good within the paediatric population, which turns it into an alternative to the traditional anticholinergics for the treatment of OB.

KEY WORDS: Overactive bladder. Tolterodine. Chilhood.

*Trabajo realizado sin ningún soporte de la industria farmacéutica. 
$\mathrm{T}$ anto en la edad adulta como en la infancia se pueden producir trastornos funcionales del aparato urinario inferior. Su clasificación, diagnóstico y tratamiento ha sido motivo de amplias controversias.

$\mathrm{Al}$ inicio se agruparon bajo el rótulo de "vejiga neurógena no neurógena”, acuñado por T. Allen en $1977^{1}$.

A pesar de que un mejor conocimiento de las entidades, sobre todo a partir de estudios urodinámicos en el momento actual, aún subsisten dudas de nomenclatura y la mayoría de autores anglosajones las engloban en "disfunciones miccionales" (voiding dysfunctions), término que consideramos incorrecto. El $2^{\text {nd }}$ International Consultation on Incontinence (julio 2001) no logra clarificar esta situación.

Preferimos englobar todos estos trastornos bajo la denominación de "trastornos funcionales no neurógenos” o mejor "disfunciones no neurógenas del aparato urinario inferior".
Entre ellos hay que distinguir las que son disfunciones predominantemente de llenado y las que lo son de vaciado (Fig. 1).

Dentro de las disfunciones de llenado, encontramos la vejiga hiperactiva (antes denominada inestabilidad vesical) y la hipoacomodación que puede simular inestabilidad.

Las disfunciones de vaciado o disfunciones miccionales, a su vez pueden agrupar diferentes cuadros:

A. Micción incoordinada o disinergia no neurógena.

B. Vejiga "perezosa" (lazy bladder).

La micción incoordinada o disinergia puede ser de detrusor y esfínter estriado. A su forma congénita se le ha llamado síndrome de Ochoa, cuando se asocia a rasgo facial característico (síndrome urofacial). La forma adquirida puede ser leve o grave, habiéndose conocido esta última como sindrome de Hinman ${ }^{2}$.

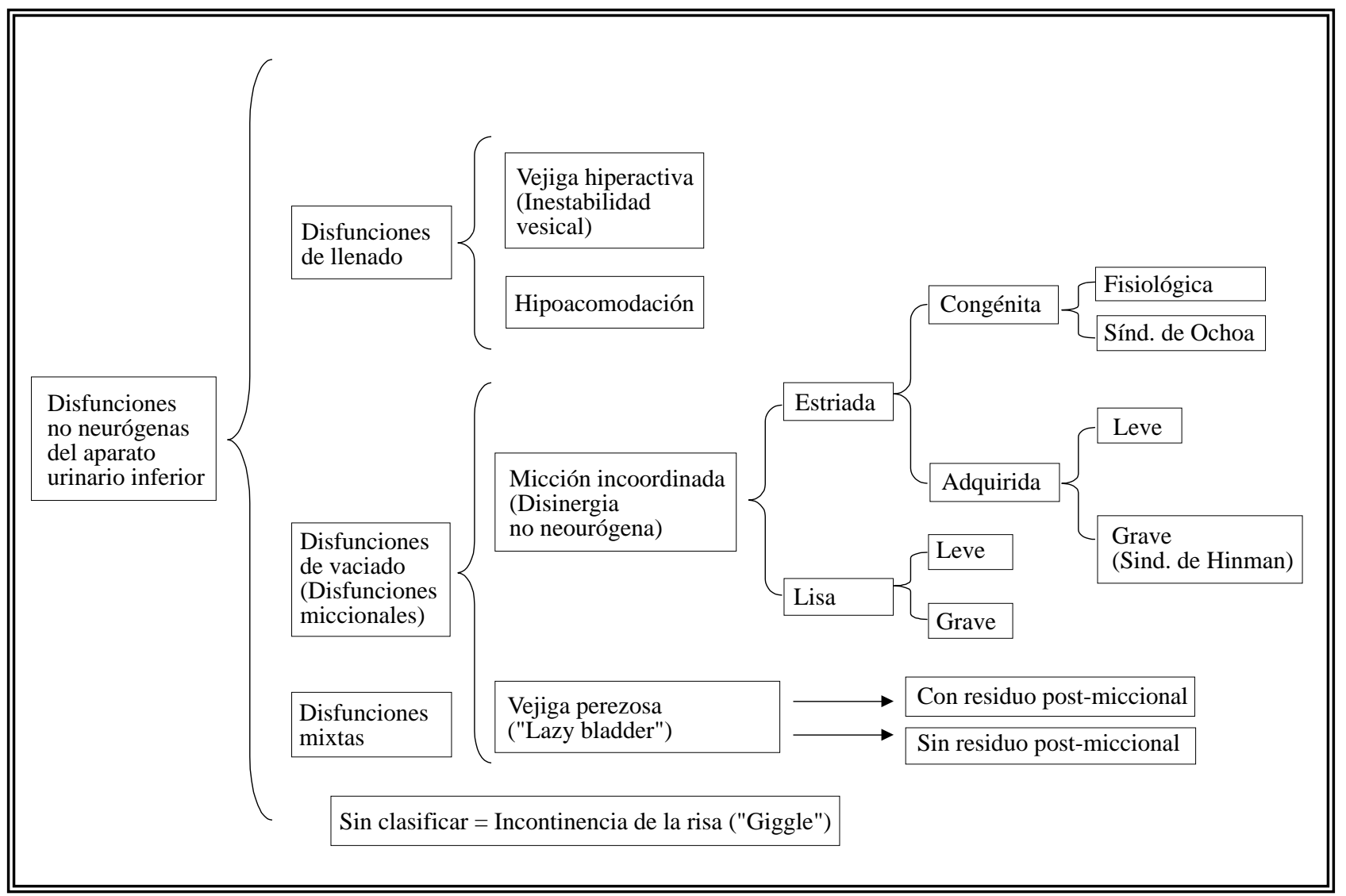

FIGURA 1. Nueva clasificación propuesta por los autores de las disfunciones no neurógenas del aparato urinario inferior. 
La disinergia puede producirse también entre detrusor y esfínter liso (disinergia lisa) siendo esta eventualidad más rara. La entidad conocida como "vejiga perezosa" está menos definida y puede cursar con o sin residuo post-miccional.

Esta clasificación intenta sistematizar estas entidades. Sin embargo, no podemos desconocer la existencia de cuadros mixtos, que asocian componentes de más de una. Los tratamientos más frecuentes se esquematizan en la Figura 2.
En el presente trabajo nos centraremos en el tratamiento de la forma más corriente de disfunción no neurógena: vejiga hiperactiva (VHA) o inestabilidad vesical. Su frecuencia es muy alta ya que la padece, según Abrams et al. ${ }^{3}$ aproximadamente el $10 \%$ de la población. Su prevalencia en niños es desconocida. Según Reinberg et al. ${ }^{4}$ tendrian sintomas de hiperactividad el $15 \%$ de los niños a los 6 años. Se considera que un patrón de vejiga o detrusor hiperactivo sería fisio-

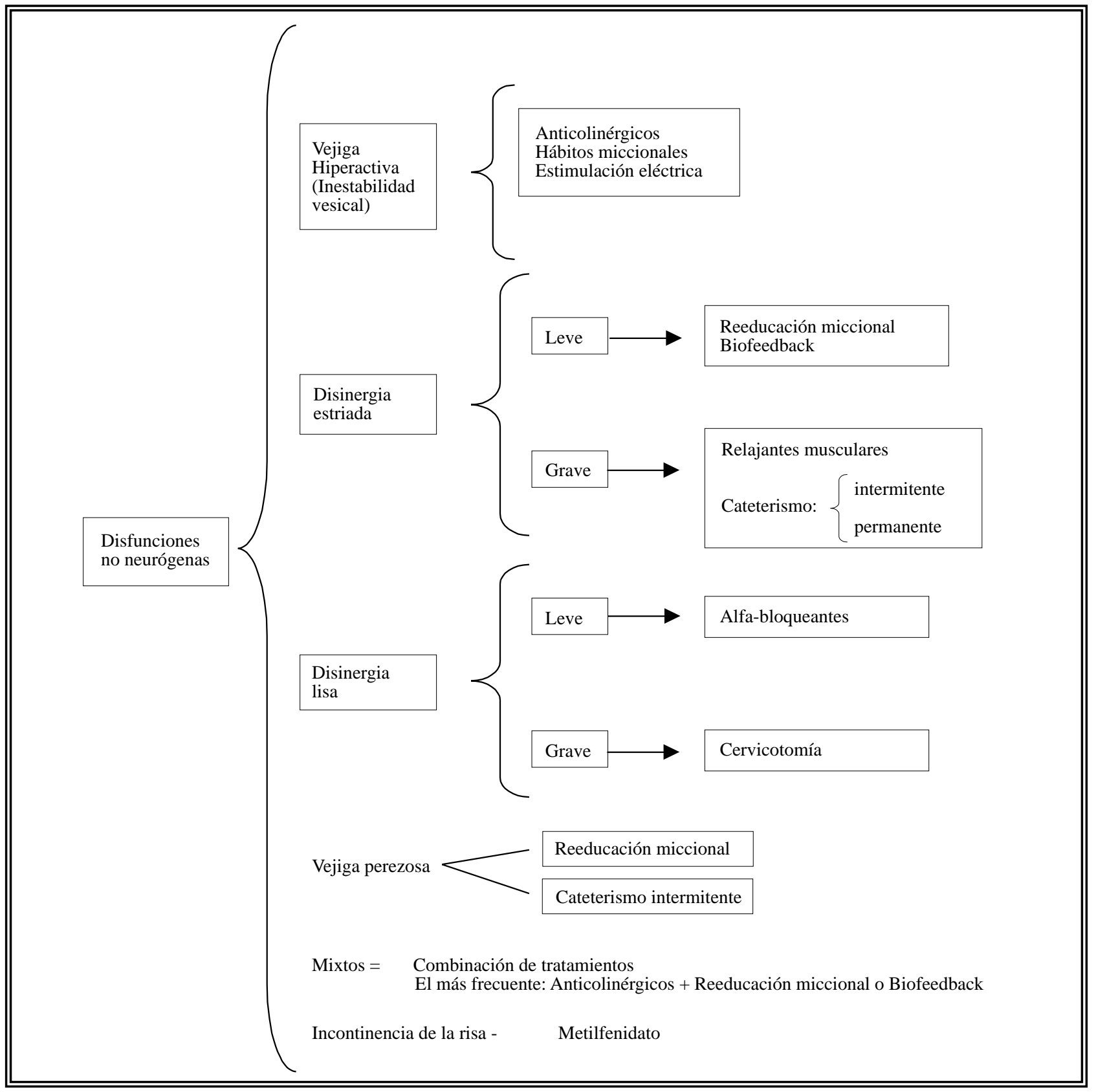

FIGURA 2. Esquema de tratamiento. 
lógico hasta alrededor de los 3 años. La combinación de la VHA con otras entidades es una realidad, aunque aún desconocemos la totalidad de su mecanismo de interrelación.

La imperiosidad con o sin polaquiuria, la incontinencia de urgencia, asociados o no a enuresis nocturna, son fácilmente correlacionables con VHA. Las contracciones "no inhibidas" durante la fase de llenado, obligan a micciones frecuentes o se produce incontinencia.

Más difícil es correlacionar fisiopatológicamente la VHA con el RVU o las infecciones urinarias recidivantes. Sin embargo, es irrefutable su frecuente asociación. Hoebke ${ }^{5}$ sitúa esta relación en un $15 \%$.

El tratamiento fundamental de la VHA es mediante fármacos anticolinérgicos. Desde que se comenzó a emplear el cloruro de oxibutinina, éste ha pasado a ser el fármaco de referencia. $\mathrm{Si}$ bien es cierto que los niños experimentan menos efectos secundarios adversos que los adultos, constituyen a veces un motivo de interrupción del tratamiento. La dosificación cada 8 horas (3 veces al día) también es un factor de incumplimiento del tratamiento.

Desde el año 2000 hemos comenzado a utilizar un fármaco, la tolterodina, del que aún hay poca experiencia en edad pediátrica. Es un antagonista de los receptores muscarínicos con selectividad para la vejiga urinaria. Su dosificación puede realizarse cada 12 horas ( 2 veces al día) y esto facilita el cumplimiento del tratamiento.

\section{OBJ ETIVOS}

Demostrar la eficacia y tolerabilidad de tolterodina en niños con VHA, comprobada urodinámicamente.

\section{MATERIAL Y MÉTODOS}

Es un trabajo retrospectivo, observando la evolución de 72 niños que desde mayo de 2000 a marzo de 2003, fueron tratados con tolterodina.

Todos tenían una vejiga hiperactiva no neurógena, diagnosticada mediante cistomanometría (contracciones involuntarias en la fase de llenado de intensidad superior a $15 \mathrm{~cm} \mathrm{H20}$ ).

Las edades oscilaban de los 5 a los 17 años (media: 10,9); 43 eran niños y 29 niñas.

Los motivos de consulta quedan reflejados en la Tabla I.

\section{TABLA I}

- Enuresis nocturna más sintomas diurnos (escapes, imperiosidad, polaquiuria).

- Enuresis nocturna y tenían más de 12 años.

- Síntomas diurnos: urgencia miccional (imperiosidad) y/o polaquiuria.

- Escapes intermiccionales diurnos.

- Infecciones urinarias de repetición

- Reflujo vésico-ureteral diagnosticado.

Ninguno tenía infección urinaria en el momento del inicio del tratamiento.

En ningún caso había elementos para sospechar que tuvieran una vejiga neurógena: ninguno tenía antecedentes, fueron explorados neurológicamente en nuestra consulta y se realizó de forma protocolizada ecografía y radiografía simple de columna, que no mostraron elementos para sospechar neurogenicidad. Sin embargo, no fueron explorados por neurólogo ni se realizó sistemáticamente resonancia magnética nuclear (RMN) de columna.

Las ecografías fueron normales exceptuando vejiga de paredes engrosadas (23 casos claros, 12 casos dudosos por repleción incompleta en el momento de la exploración). Los niños con reflujo tenían anomalías renales variadas.

Ninguno de los niños tenía patología capaz de provocar incontinencia de origen orgánico (ejemplo: uréter de desembocadura ectópica, epispadias, etc.).

Ninguno había sido tratado antes con anticolinérgicos. Los niños enuréticos tampoco estaban recibiendo tratamiento con Desmopresina (por lo menos tres meses antes).

A todos se realizó un EUD previo: flujometría y cistometría. Todos tenían elementos cistomanométricos que configuraban un claro cuadro de VHA y el flujo urinario estaba dentro de los límites normales.

Así como consideramos imprescindible la realización de EUD dentro de los criterios clínicos especificados en la Tabla I, antes de iniciar el tratamiento, dependiendo de un gran número de factores (mejoría clínica evidente, negativa de los padres, dificultad de realización del primer estudio, etc.), la práctica de un nuevo EUD es más variable. 
En nuestros pacientes establecimos dos grupos de estudio:

Grupo A: Niños en los que se realizó EUD pre y post-tratamiento.

Grupo B: Niños en los que se realizó EUD pretratamiento exclusivamente.

\section{Grupo A: 27 niños}

- Diagnosticados de reflujo vésico-ureteral (12 niños). Se les practicó EUD por:

Signos asociados en la cistografía que sugerían vejiga hiperactiva.

Engrosamiento de paredes vesicales en la ecografía.

Infecciones recidivantes a pesar de correcta quimioprofilaxis.

Indicación de corrección quirúrgica del reflujo.

- Pacientes con infecciones urinarias recidivantes (15 niños).

En la cistomanometría inicial se comprobó VHA. Se realizó nuevo EUD entre 5 y 8 meses después.

\section{Grupo B: 45 niños}

Consultaron por síntomas diurnos (urgencia, polaquiuria, escapes), síndrome enurético o enuresis nocturna con 12 años o más.

En todos se comprobó urodinámicamente VHA.

Se evaluaron clínicamente entre 6 y 12 meses de iniciado el tratamiento.

La dosis de Tolterodina fue siempre la misma: $2 \mathrm{mg}$ vía oral, dos veces al día, por períodos que oscilaron entre los 4 y los 13 meses.

Se advertía a los padres que en el prospecto del medicamento leerían: "No se han determinado todavía la seguridad ni la eficacia en los niños" pero que había suficientes trabajos experimentales que nos permitían indicar la medicación en niños mayores de 5 años.

El seguimiento mínimo fue de 4 meses y el máximo de 31 .

\section{RESULTADOS}

Grupo A

En el segundo EUD, realizado entre 5 y 8 meses de iniciado el tratamiento, se comprobó:
- Curación (desaparición del cuadro de hiperactividad vesical) en un $67 \%$ de los pacientes.

- Mejoría (menor número de contracciones y/o contracciones de menor presión) en un 14\%.

- Urodinámicamente sin cambios en un 19\%.

\section{Grupo B}

De acuerdo a los criterios de la International Children's Continence Society (ICCS) ${ }^{6}$ se consideró:

- Curación: $>90 \%$ de mejoría de síntomas

- Mejoría 50-90\% de mejoría de síntomas

- Fallo $<50 \%$ de mejoría de síntomas

Con un tratamiento de entre 5 y 13 meses, nuestros resultados fueron:

- Curación: $51 \%$

- Mejoría: 27\%

- Fallo: $22 \%$

No encontramos diferencias significativas entre los dos sexos en cuanto a resultados, tanto en los niños del grupo A como en los del grupo B. Tampoco encontramos diferencias relacionadas con la edad.

El porcentaje de efectos adversos fue muy bajo, pero imposible de cuantificar por falta de referencia a los mismos en todas las historias clínicas. Sólo citaremos los más frecuentemente comentados: sequedad de boca, visión lejana borrosa, picor en los ojos, enrojecimiento de meji1las. Dos niños refirieron cefaleas de escasa entidad; uno un episodio de epistaxis. Ninguno refirió trastornos gastrointestinales. No hay constancia de ningún efecto secundario grave.

En ningún caso fue necesario suspender la medicación por dichos efectos secundarios.

\section{DISCUSIÓN}

La causa de la VHA no neurógena es desconocida $^{7}$. Como factores etiológicos en niños se han invocado: retardo madurativo, prolongación del comportamiento infantil de la vejiga, errores en la fase de educación esfinteriana y factores hereditarios.

La VHA ha sido correlacionada con otras patologías en la infancia. La interrelación entre VHA y $\mathrm{RVU}^{5,8,9}$ no está suficientemente aclarada. Se supone $^{5}$ que la hipertrofia del detrusor y las altas presiones en la fase de llenado, inciden en el mantenimiento del reflujo. Existe la idea ${ }^{5}$ de que, 
corrigiendo la inestabilidad, se contribuye a propiciar la desaparición del reflujo. Sin embargo, no hay datos de ello basados en la evidencia.

La mayor frecuencia de infección urinaria en los niños con VHA es una realidad no bien explicada. La reintroducción, por un proceso "aspirativo" de orina que ha llegado al sector distal de la uretra en la niña, podría ser un mecanismo ${ }^{5}$.

La asociación con estreñimiento ${ }^{5-10}$ contribu- $^{-}$ ye a una mayor virulencia de la flora contaminante fecal.

Los síntomas característicos de la VHA son polaquiuria (mayor o igual 8 micciones en 24 horas) $^{3}$, imperiosidad e incontinencia de urgencia. Su efecto nocivo en cuanto a la calidad de vida del niño es evidente. Puede cursar con un síndrome enurético.

A nuestro juicio no basta con sospechar una VHA para iniciar un tratamiento farmacológico, muchas veces, prolongado. Hemos observado, entre los padres de nuestros pacientes una frecuente reticencia a iniciar un tratamiento largo, con posibilidad de ciertos efectos adversos, sin tener una evidencia como la urodinamia. No obstante, hay muchos autores que piensan que una exploración invasiva no está definitivamente justificada $^{4,11,12}$.

Varios fármacos anticolinérgicos han sido utilizados en el tratamiento de la VHA. El más frecuente y con el que hay una más larga experiencia es el cloruro de oxibutinina ${ }^{13,14}$. Su dosificación se ha de realizar con tomas tres veces al día, lo que hace menos asumible el cumplimiento por parte del niño (sobre todo, por la toma del mediodía que coincide con el horario escolar).

$\mathrm{El}$ cloruro de trospio parece tener menos efectos secundarios que la oxibutinina ${ }^{15}$ y similar efectividad terapéutica. Recientemente se ha realizado en España un ensayo clínico multicéntrico randomizado, simple ciego frente a placebo, en niños. Nuestra Unidad participó en él y los resultados son alentadores ${ }^{16}$.

Otros tratamientos han sido utilizados para la VHA de la infancia: biofeedback, reeducación miccional con métodos conductistas, estimulación eléctrica ano-genital y neuromodulación ${ }^{8,10,17-20}$. Sus resultados aún son inciertos y está pendiente la demostración de sus beneficios en niños.
Desde 1997 se vienen publicando trabajos sobre un antimuscarínico de acción selectiva sobre la vejiga: la tolterodina. Tiene una más específica acción sobre ésta que sobre las glándulas salivares ${ }^{21}$.

Se han publicado estudios en fase II y $\mathrm{III}^{3}$. El primer trabajo en niños es el de Goessl ${ }^{22}$. Se trata del uso de este fármaco en niños con vejiga neurógena hiperrefléxica. Este primer estudio de eficacia y tolerancia en población pediátrica, arrojó resultados interesantes comprobados urodinámicamente, con efectos adversos escasos y leves.

En 2001 ya se publicaron ensayos clínicos ${ }^{12}$ y estudios retrospectivos ${ }^{23}$ en VHA no neurógenas.

Hjälmas et al. ${ }^{12}$ realizan un ensayo para averiguar seguridad, eficacia y dosis óptima. Los efectos secundarios son notoriamente menores, atribuyéndose este hecho a la baja lipofilia del fármaco ( 8 veces menor potencia antimuscarínica sobre la parótida que la oxibutinina). Su vida media es más larga, por lo que se puede dosificar en dos tomas diarias. Estos hechos contribuyen a un mejor cumplimiento del tratamiento. La dosis recomendada por Hjälmas et al. ${ }^{12}$ fue de 1 $\mathrm{mg}$ dos veces al día. Munding et al. ${ }^{23}$ realizan un estudio retrospectivo en 30 niños, aplicando nuestros mismos criterios de valoración de los resultados, pero sin EUD previo. Ellos piensan que esta exploración no es imprescindible ya que su correlación con la clínica es de un $80 \%$. Sus resultados son similares a los nuestros.

En los últimos tiempos se ha difundido, sobre todo en EE.UU, el uso de anticolinérgicos de acción prolongada $^{4-24}$. Quizás signifiquen un avance por mejor respuesta y mayor cumplimiento.

\section{CONCLUSIONES}

El análisis de nuestra casuística demuestra unos buenos resultados tanto clínicos como urodinámicos. Quizás sería necesario esperar algo más de tiempo para analizar estos resultados con más perspectiva y también poder observar lo referido en la literatura consultada: recidivas del cuadro de VHA.

La tolerancia y efectividad de Tolterodina en VHA en los niños es buena. Los efectos adversos que provoca son escasos y de poca entidad clínica. Los niños, en general, toleran mejor que los adultos los efectos farmacológicos secundarios de los anticolinérgicos. 
Creemos muy útil la realización de un estudio urodinámico que confirme la VHA, ya que la aceptación y cumplimiento del tratamiento será mejor, como lo demuestra nuestro estudio retrospectivo.

\section{REFERENCIAS}

1. ALLEN T.: The non neurogenic-neurogenic bladder. J Urol 1977; 117: 232-234.

2. HINMAN F, BAUMAN F.: Vesical and ureteral damage from voiding dysfunction in boys without neurologic or obstructive disease. J Urol 1973; 109: 727-732.

3. ABRAMS P, FREEMAN R, ANDERSTRÖM C, MATIASSON A.: Tolterodine, a new antimuscarinic agent: as effective but better tolerated than oxybutynin in patients with an overactive bladder. BJU Int 1998; 81: 801-810.

4. REINBERG Y, CROCKER J, WOLPERT J, VADERSTEEN D.: Therapeutic efficacy of extended release oxybutynin chloride and immediate release and long acting tolterodine tartrate in children with diurnal urinary incontinence. $J$ Urol 2003; 169: 317-319.

5. HOEBKE P, VANDEWALLE J.: Voiding dysfunction, recurrent urinary tract infections, constipation a vesico-ureteral reflux: a common disease complex. In: current management of dysfunctional voiding. Dial in Pediatr Urol 2002; 25: 8.

6. NORGAARD J, VAN GOOL J, HJÄLMAS, K, DJURHUUS J, HELLSTROM A.: Standarization and definitions in lower urinary tract dysfunction in children. BJU 81 1998; Suppl 3: 1-16.

7. GRENFIELD S.: Editorial: the overactive bladder in childhood. J Urol 2000; 163: 578-579.

8. CURRAN M, KAEFER M, PETERS C, LOGIGIAN E, BAUER S.: The overactive bladder in childhood: long-terms results with conservative management. J Urol 2000; 163: 574-577.

9. VEGA J, PASCUAL L.: High-pressure bladder: an underlying factor mediating renal damage in absence of reflux? BJU Int 2001; 87: 581-584.

10. WIENER J, SCALES M, HAMPTON J, KING L, SURWIT R, EDWARDS C.: Long-term efficacy of simple behavioral therapy for daytime wetting in children. J Urol 2000; 164: 786-790.

11. COOPER C.: Current management of dysfunctional voiding. Dial in Pediatr Urol 2002; 25: 8.

12. HJÄLMAS K, HELLSTROM A, MOGREN K, LACKGREN G, STENBERG A.: The overactive bladder in children: a potential future indication for tolterodine. BJU Int 2001; 87: 569-574.
13. GARAT J.: Tractament farmacologic de l'enuresi. But Soc Cat Pediatr 1991; 51: 121-126.

14. GARAT J, CAFFARATTI J.: Diagnóstico y tratamiento farmacológico de la enuresis funcional y monosintomática. Urol Integr Invest 1996; 1: 113118.

15. OSCA-GARCÍA J, MARTÍNEZ E, CONEJERO J, JIMÉNEZ F.: Cloruro de trospio versus oxibutinina en el tratamiento de la vejiga hiperactiva. Urod Aplic 1997; 10: 40-43.

16. LÓPEZ-PEREIRA P, MIGUELEZ C, CAFFARATTI J, ESTORNELL F, ANGUERA A.: Trospium chloride in the treatment of detrusor instability in children: a single blind randomized, multicentre, placebo controlled study. $J$ Urol (en prensa).

17. YAMANISHI T, YASUDA K, MURAYAMA N, SAKAKIBARA R, UCHIYAMA T, ITO H.: Biofeedback training for detrusor overactivity in children. $J$ Urol 2000; 164: 1686-1690.

18. AUSTIN J, SCHULMAN S.: Treatment of dysfunctional voiding with biofeedback. Dial in Pediatr Urol 2002; 25: 8.

19. BOWER W.: Neuromodulation as an adjunct to inervation for voiding dysfunction in children. Dial in Pediatr Urol 2002; 25: 8

20. GLADH G, MATTSSON S, LINDSTRÖM S.: Anogenital electrical stimulation as treatment of urge incontinence in children. BJU Int 2001; 87: 366371.

21. MESSELINK E.: Treatment of the overactive bladder with tolterodine, a new muscarinic receptor antagonist. BJU Int 1999; 83: 48-52.

22. GOESSL C, SAUTER T, MICHAEL T, BERGE B, STAEHLER M, MILLER K.: Efficacy and tolerability of tolterodine in children with detrusor hyperreflexia. Urology 2000; 55: 414-418.

23. MUNDING M, WESSELLS H, THORNBERRY B, RIDEN D.: Use of tolterodine in children with dysfunctional voiding: an initial report. J Urol 2001; 165: 926-928.

24. VAN KERREBROECK P, KREDER K, JONAS U, ZINNER N, WEIN A.: Tolterodine once-daily: superior efficacy and tolerability in the treatment of the overactive bladder. Urology 2001; 57: 414-421.

\author{
Dr. J.M. Garat Barredo \\ Unidad de Urología Pediátrica \\ Servicio de Urología. Fundació Puigvert \\ C/ Cartagena, 340-350 \\ 08025 Barcelona
}

(Trabajo recibido el 31 julio de 2003) 